\title{
Análisis del desarrollo regional endógeno a través de la educación superior intercultural
}

\section{Analysis of endogenous regional development through intercultural higher education}

GAZCA-HERRERA, Luis Alejandro ${ }^{* *}$, GARIZURIETA-BERNABE, Jessica, GARIZURIETAMEZA, Miguel Hugo y GÓMEZ-CABAÑAS, José Rafael

Universidad Veracruzana

ID 1 ${ }^{\mathrm{er}}$ Autor: Gazca-Herrera, Luis Alejandro / ORC ID: 0000-0001-7637-2909, CVU CONACYT ID: 105089

ID $1^{\mathrm{er}}$ Coautor: Garizurieta-Bernabe, Jessica / ORC ID: 0000-0002-1443-4737, CVU CONACYT ID: 273881

ID $2^{\text {do }}$ Coautor: Garizurieta-Meza, Miguel Hugo / ORC ID: 0000-0002-1968-5121, CVU CONACYT ID: 476711

ID $3^{\text {er }}$ Coautor: Gómez-Cabañas, José Rafael / ORC ID: 0000-0002-2411-9669

DOI: $10.35429 / J U M .2020 .11 .4 .17 .26$

Recibido 12 de Abril, 2020; Aceptado 30 de Junio, 2020

\section{Resumen}

Se sostiene que el desarrollo plantea importantes retos que afectan a futuro, la equidad y las necesidades de las personas. La educación constituye una herramienta fundamental para revertir las desigualdades. En esta investigación realizada en la Universidad Veracruzana Intercultural tuvo como objetivo analizar el impacto regional endógeno desde las variables educativas interculturales; la investigación se diseñó de forma transaccional descriptiva, puesto que, a través de la evaluación de las variables definidas en torno a las dimensiones: económica, social y ambiental; se buscó describir la medida en que cada una se ha visto afectada a partir de la implementación de la Universidad Intercultural en la región de la Huasteca. El resultado muestra que la Universidad Intercultural se encuentra en proceso de cumplimiento de los ámbitos que se mencionan dentro del desarrollo endógeno, dando un impulso a la economía, a los ámbitos socio - culturales y ambientales.

Palabras clave: Desarrollo Endógeno, Educación Superior, Interculturalidad

\begin{abstract}
Development, it is argued, poses important challenges that affect the future, equity and people's needs. Education is a fundamental tool to reverse inequalities. In this research carried out at the Veracruzana Intercultural University, the objective was to analyze the endogenous regional impact from the intercultural educational variables; The research was designed in a descriptive transactional way, since, through the evaluation of the variables defined around the dimensions: economic, social and environmental; An attempt was made to describe the extent to which each one has been affected by the implementation of the Intercultural University in the Huasteca region. The result shows that the Intercultural University is in the process of fulfilling the areas mentioned within endogenous development, giving a boost to the economy, to socio-cultural and environmental areas.
\end{abstract}

Endogenous Development, Higher Education, Interculturality

Citación: GAZCA-HERRERA, Luis Alejandro, GARIZURIETA-BERNABE, Jessica, GARIZURIETA-MEZA, Miguel Hugo y GÓMEZ-CABAÑAS, José Rafael. Análisis del desarrollo regional endógeno a través de la educación superior intercultural. Revista de Gestión Universitaria. 2020. 4-11: 17-26

\footnotetext{
* Correspondencia del Autor (Correo electrónico: lgazca@uv.mx)

$\dagger$ Investigador contribuyendo como primer autor.
} 


\section{Introducción}

Las circunstancias históricas, políticas, sociales y culturales confluyen en los retos y posibilidades para el nivel de desarrollo de una nación. El desarrollo, es un concepto socialmente construido que varía de una sociedad a otra, sin embargo, encierra la idea de progreso. Todas las sociedades del mundo aspiran a progresar. Si bien es cierto, que el mundo ha progresado más en los últimos años, también la brecha de desigualdad ha incrementado (Schwartzman, 2005). Partiendo de la relación que existe entre la educación y el desarrollo; y con base a en un enfoque endógeno; la presente investigación realizó un análisis sobre el impacto que ha tenido la Universidad Veracruzana Intercultural en las dimensiones económica, social y medioambiental -desde una visión más estructural que coyuntural-, en la región de la Huasteca del Estado de Veracruz.

Es importante mencionar que uno de los factores que más influyen en el desarrollo de un país es la educación, a través de los años ha sido considerada necesaria e importante para el desarrollo económico del mismo (Muñoz, 2014). Para entender el desarrollo en términos del impacto regional económico de un país es importante conocer las necesidades que tiene su población, y comprobar si estas están satisfechas. Existen cuatro grandes teorías que los sustentan: modernización, dependencia, sistemas mundiales y globalización, mismas que ayudan a interpretar teóricamente el nivel económico en el que se encuentran las naciones. La primera teoría que sustenta el desarrollo económico es la de la modernización, la cual supone una visión de desarrollo integral, es decir, combinas variables de economía, política, sociología, cultura e historia para lograrlo, resaltando la importancia del papel de las instituciones y sus relaciones con el estado (Kotler, 1998).

"Se establece que las sociedades modernas son más productivas si los niños están mejor educados" (Kotler, 1998), derivado de ello resalta la prioridad que se le debe dar a la educación, no sólo a los niños sino hasta la edad adulta; puesto que la ciencia produce conocimiento, la educación lo transmite y después de recibirla el deber ser del estudiante es aplicarla en la vida real, por ende, entre mayor sea el grado de educación, mejores serán los resultados de la productividad obtenida.
La teoría de la modernización surge en la década de los 50’s y 60’s como respuesta a la batalla ideológica entre el capitalismo y el comunismo considerados como una amenaza regional, un mal ejemplo para los países tercermundistas (Bula, 1994). Fue Walt Whitman Rostow, principal autor de la teoría de la modernización, quien sostenía que el desarrollo económico era un proceso de múltiples estados, estimulado por el deseo de mejorar la vida y lograr beneficios, así el proceso de desarrollo sólo se logra a través de etapas que iniciaban con un rápido despegue producido por el crecimiento estimulado de los principales sectores de la economía (Tito, 2012).

Existe una relación directa entre la educación superior y el deseo de mejorar la vida diaria, puesto que se ha manejado a través de los años que entre mayores sean los grados de estudio obtenidos mayor será la remuneración y en consecuencia, se contribuye al desarrollo de una sociedad; cuando no se tiene la ambición por obtener estos beneficios, o bien, los medios no son los adecuados para conseguir el progreso, se vive un periodo de estancamiento, donde la modernización sólo es percibida por ciertos sectores de la población.

Con base en los párrafos anteriores, se entiende que la modernización es el proceso que llevan las sociedades tradicionales hacia la modernidad y que se refleja en una serie de cambios generales: urbanización, industrialización, secularización, racionalidad, diferenciación social, aumento del alfabetismo, extensión de los medios de comunicación, mayor control del entorno natural y social, crecimiento económico, una más compleja división del trabajo, un desarrollo político expresado en mayor movilización social y mayor participación política. Siendo estas las principales características del desarrollo de la modernidad de una sociedad (Huntington, 2006).

Como un intento de explicar la situación de estancamiento socioeconómico de Latinoamérica durante el siglo XX, surge entre la década de los 50's y 70's la teoría de la dependencia. De acuerdo con Blacutt (2013), en esta teoría se proponía que el mundo se divide en dos clases de países: los del centro y los de la periferia. 
De acuerdo con la "Ley de la Ventaja Comparativa", los primeros producen bienes de alto valor agregado y los segundos deben contentarse con la producción de materias primas. Esta asimetría en la división internacional del trabajo da por resultado una relación de términos de intercambio en el comercio internacional, la que sería desfavorable a los países subdesarrollados.

Ahora bien, la teoría del desarrollo endógeno comprende todas las mejoras que se pueden presentar en una organización, tiene que ver con la proyección y utilización de las capacidades de los individuos en relación con la función creativa y emprendedora que pudieran tener los habitantes de una comunidad. La dimensión del desarrollo endógeno económico se sostiene sobre un conjunto de relaciones de producción, transformación, distribución y consumo de medios materiales y económicos que se obtienen a partir de la acción de las personas (Delgado, 2010). Por lo tanto, se puede decir que el desarrollo endógeno es aquella teoría que afirma que la sostenibilidad de una economía es de adentro hacia afuera, es decir, si dentro de una comunidad se potencian sus habilidades y capacidades en los diversos ámbitos como cultural, moral, social, político y tecnológico, será posible el fortalecimiento y madurez de ésta, contribuyendo externamente a su crecimiento y desarrollo: "Esto supone una propuesta de futuro para la cultura, para la economía, para la ética, para la tecnología, etc., hasta llegar a poder consolidar una imagen objetivo integral de comunidad, nación o sociedad, y que el desarrollo endógeno proponga como fin y como futuro alcanzable" (Morán, 2007).

\section{Educación Intercultural}

En el ámbito de la educación superior intercultural, con el objetivo de promover la perspectiva de la acción intercultural en la educación superior, durante la mitad de la primera década del siglo XXI, surgió la "Universidad intercultural". De tal forma que, se puede concebir como un espacio "donde los miembros de diferentes culturas se relacionan entre sí en un marco de respeto" (Romero Ávila, 2011: 24). En el sexenio del presidente Vicente Fox (2000-2006), se crean las universidades interculturales, dichas instituciones se ubicaron en regiones con alta densidad indígena.
En un principio cada Universidad veía desde su particular perspectiva los ejes de formación, dando como resultado la generación "de propuestas disímbolas sobre la misma carrera, que en algunos casos eran verdaderamente contrastantes" (Ávila Romero \& Ávila Romero, 2016: 207). No fue sino hasta el 2011, que se comenzaron una serie de reuniones con el objetivo de analizar los mapas curriculares y favorecer su conjunción en un programa académico unitario, dando como resultado que en la actualidad se cuente con nueve instituciones de educación superior con orientación intercultural, ocho de las cuales responden directamente al modelo educativo intercultural, la otra, la Universidad Autónoma Indígena de México, se creó en el año 2000 y es afín a las Universidades Interculturales.

Las ocho restantes se crearon entre los años 2003 y 2007 y son, según orden de antigüedad, la Universidad Intercultural del Estado de México (creada en el año 2003), la Universidad Intercultural de Chiapas (2004), la Universidad Veracruzana Intercultural (2004), la Universidad Intercultural del Estado de Tabasco (2005), la Universidad Intercultural del Estado de Puebla (2006), la Universidad Intercultural Indígena de Michoacán (2006), la Universidad Inter- cultural Maya de Quintana Roo (2007) y la Universidad Intercultural del Estado de Guerrero (2007). (Guitart y Rivas, 2008: 156).

De acuerdo con Muñoz (2014), el impacto de la educación en el desarrollo de México ha sido escasa para los sectores populares de la población, alcanzando quizá adquirir algunos grados de educación primaria, aún con ello, existe mucho desempleo de la fuerza de trabajo. En suma, aunque la educación intercultural busca contribuir a lograr sociedades más justas, productivas y equitativas; y en consecuencia influir en el desarrollo económico del país, aún hay acciones que deben ser revisadas.

\section{Marco Referencial}

La Universidad Veracruzana Intercultural (UVI) región de la Huasteca, se localiza en la parte norte del Estado de Veracruz, formando parte de la llanura costera del Golfo de México. Está conforma por 33 municipios, los cuales abarcan una superficie de 20,239.08 $\mathrm{Km} 2$, correspondiente al $26.25 \%$ de la superficie total del estado de Veracruz (figura 1). 


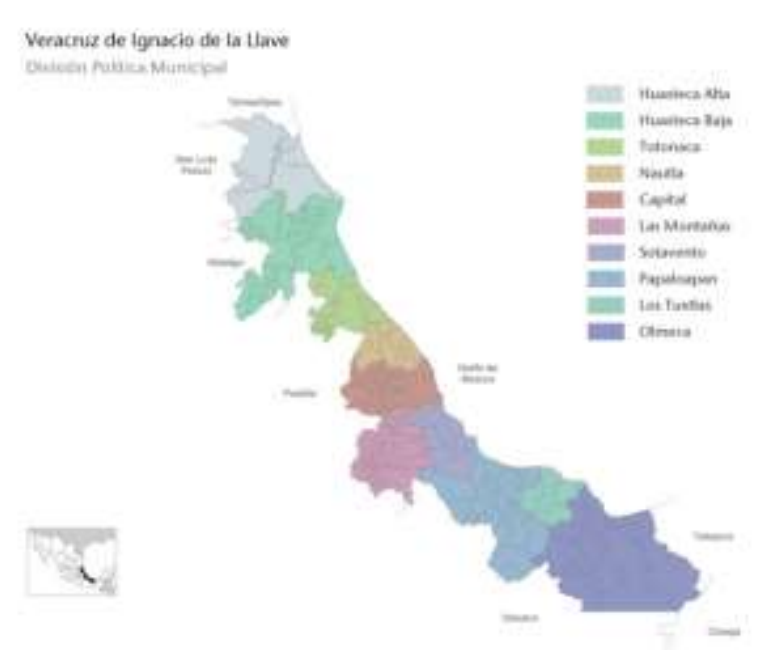

Figura 1 Región Huasteca

Fuente: (Polanco, 2012)

La población total en esta zona es de 975,207 habitantes, y de los cuales se tienen registrados 306,795 habitantes como población económicamente activa, distribuidos en tres sectores:

El sector primario, donde se llevan a cabo actividades de agricultura, ganadería, caza y pesca, le corresponde el $42.64 \%$ de la población ocupada.

El sector secundario, que abarca las actividades de minería, extracción de petróleo y gas natural, industria manufacturera, electricidad, agua y construcción, registra en esta zona el $18.99 \%$ de la población ocupada.

El sector terciario, con actividades como el comercio, transporte y comunicaciones, servicios en general, así como profesionales y técnicos, restaurantes, hoteles, personal de mantenimiento y otros), el $36.46 \%$ de la población se ocupa en este sector.

\begin{tabular}{|c|c|}
\hline Sede & Municipios \\
\hline Huasteca & $\begin{array}{l}\text { Ixcatepec, Chontla, } \\
\text { Tepetzintla, Ixhuatlán de Madtépetl, } \\
\text { Tlachichilco, Zacualpan, Texcapetec, } \\
\text { Zontecomatlán, Ilamatlán, Benito Juárez, } \\
\text { Chicontepec, Chalma, Chiconamel, Platón } \\
\text { Sánchez, Tantoyuca y otros municipios } \\
\text { veracruzanos y de estados vecinos dentro } \\
\text { de la Región Intercultural Huasteca }\end{array}$ \\
\hline
\end{tabular}

Tabla 1 Municipios que abarcan las sede Huatesca de la Universidad Intercultural

Fuente: Elaboración propia, basado en (Universidad Veracruzana, 2015)
La Universidad Veracruzana, con la finalidad de reconocer la diversidad cultural y atender las necesidades de acceso a estudios de educación superior de la población de las Regiones Interculturales, a través de la UVI ha desplegado diferentes programas para favorecer el desarrollo de acciones educativas, en diferentes niveles educativos (Universidad Veracruzana, 2015). A través de una de sus sedes regionales, se identifican los municipios que abarca la sede Huasteca (tabla 1).

Actualmente, en la sede se oferta la Licenciatura en Gestión Intercultural para el Desarrollo (LGID), dicha licenciatura se constituye de cinco Orientaciones: Lenguas, Comunicación, Sustentabilidad, Derechos y Salud. Dicho programa educativo busca fortalecer el tejido sociocultural, el buen manejo de recursos naturales, promover la salud, la participación ciudadana; con miras a un desarrollo sustentable, en términos de justicia social (Universidad Veracruzana, 2007)

\section{Metodología}

Se empleó básicamente un modelo de investigación evaluativo, mediante la aplicación de diversos métodos de investigación, que permitieron evaluar el impacto de la UVI en la región de la huasteca. El objetivo fue medir los resultados obtenidos a partir de su implementación debido a la dimensión económica, social y ambiental; propuestos por el desarrollo endógeno. Considerando el contexto que rodea a la investigación y el tipo de estudio efectuado, la investigación se diseñó de forma transaccional descriptiva, puesto que, a través de la evaluación de las variables definidas en torno a las dimensiones: económica, social y ambiental; se busca describir la media en que cada una se ha visto afectada a partir de la implementación de la UVI en la región de la huasteca.

El método que se utilizó para la selección de las unidades de muestreo fue por conglomerado, debido a que la región de estudio abarca una extensa zona geográfica. Para ello, haciendo uso de un mapa de la región de la Huasteca y considerando la ubicación de la UVI, se dividió el total de la población en tres conglomerados: Ixhuatlán de Madero $(50,902)$, Benito Juárez $(16,880)$ y Chicontepec $(55,292)$; y se tomó al azar una muestra. 
El total de la población mayor de 18 años ubicada en la región de la huasteca en base a datos del INEGI es de 123,074 habitantes. De este total, y haciendo uso de la fórmula:

$n=\frac{Z^{2} N p q}{e^{2}(N-1)+Z^{2} p q}$

En donde:

$Z=$ Nivel de confianza.

$N=$ Universo o población

$p=$ Población a favor .

$q=$ Población en contra .

$e=$ Error de estimación (precisión en los resultados).

$n=$ Número de elementos (tamaño de la muestra).

Considerando los siguientes datos:

$Z=95 \%=1.96$

$N=$ Población mayor de 18 años

$p=50 \%=0.50$

$q=50 \%=0.50$

$e=5 \%=0.05$

$\mathrm{n}=$ ?

Y sustituyendo en la formula, se obtuvo que:

$n=\frac{(1.96)^{2}(123,074)(.50)(.50)}{(.05)^{2}(123,074-1)+(1.96)^{2}(.50)(.50)}$

$n=\frac{118,200.26}{308.64}=382.97$

Se obtuvo un tamaño de muestra de 383 pobladores. A fin de tomar de acuerdo con los porcentajes con base 100, una muestra de cada conglomerado, el total del tamaño de la muestra fue dividió entre estos, de manera que se generaron 3 de muestras representativas, cada una conformada por:

158 personas correspondientes al $41.35 \%$ de la localidad de Ixhuatlán de Madero

172 personas correspondientes al $44.92 \%$ de la localidad de Chicontepec

53 personas correspondientes al $13.73 \%$ de la localidad de Benito Juárez
Tomando como objeto de estudio la participación de estudiantes y egresados de los programas educativos ofertados en la Universidad Veracruzana Intercultural sede Huasteca, así como los habitantes de las comunidades que abarcan la región de la Huasteca; para la categorización de variables se definieron tres dimensiones, con dos categorías y cuatro indicadores en cada una de ellas (Tabla 2).

\begin{tabular}{|c|c|c|}
\hline Categorías & Subcategorías & Indicadores \\
\hline \multirow[t]{2}{*}{ Económica } & Desarrollo & $\begin{array}{l}\text {-Individual - Familiar } \\
\text { - Empresarial } \\
\text { Comunitario }\end{array}$ \\
\hline & Impacto & $\begin{array}{l}\text {-Individual - Familiar } \\
\text { - Empresarial } \\
\text { Comunitario }\end{array}$ \\
\hline \multirow[t]{2}{*}{$\begin{array}{l}\text { Social- } \\
\text { Cultural }\end{array}$} & Desarrollo & $\begin{array}{l}\text {-Individual - Familiar } \\
\text { - Empresarial } \\
\text { Comunitario }\end{array}$ \\
\hline & Impacto & $\begin{array}{l}\text {-Individual - Familiar } \\
\text { - Empresarial } \\
\text { Comunitario }\end{array}$ \\
\hline \multirow[t]{2}{*}{ Ambiental } & Desarrollo & $\begin{array}{l}\text {-Individual - Familiar } \\
\text { - Empresarial - } \\
\text { Comunitario }\end{array}$ \\
\hline & Impacto & $\begin{array}{l}\text {-Individual - Familiar } \\
\text { - Empresarial } \\
\text { Comunitario }\end{array}$ \\
\hline
\end{tabular}

Tabla 2 Categorización de las Variables Fuente: Elaboración Propia (2020)

A partir de las diversas dimensiones desde las cuales se puede analizar el impacto del desarrollo endógeno, se consideraron tres categorías principales:

Económica. Analiza los aspectos económicos de la región en cuanto a capitales, inversiones, ahorros y financiamiento.

Social- cultural. Analiza la región desde el aspecto del desenvolvimiento de los habitantes de la región, sus relaciones, su forma de vida, sus raíces, etc.

Ambiental. Analiza los daños ecológicos y ambientales de la región, así como las medidas tomadas para reparar los daños. 
A su vez, cada una de ellas contiene dos subcategorías que representan los principales avances que se observan en la región: la primera es el desarrollo, la cual se refiere al proceso de transformación que se ha presentado después de la llegada de la UVI a la región de la Huasteca, así como la forma de participación en los procesos y decisiones a nivel operativo y estratégico de la comunidad; la segunda es el impacto, el cual es medido a raíz de los cambios que se han presentado a través del tiempo, tanto en las estructuras externas como internas. Y, por último, cada variable se derivan cuatro indicadores, los cuales hacen referencia de los niveles en los cuales se presentan los avances dentro de cada dimensión, estos son:

El personal, el cual se refiere a aquel individuo perteneciente a la localidad que se estudia que ha sido favorecido con la colaboración de la Universidad Veracruzana Intercultural;

El familiar, referido al grupo de personas que han sido participes directos $\mathrm{o}$ indirectos del proyecto de la Universidad Veracruzana;

El empresarial, el cual considera a aquellas personas que tienen a su cargo una unidad con fines económicos y que su papel ha sido activo con la comunidad universitaria;

El comunitario, donde participan todos aquellos miembros de la región que en conjunto se han visto beneficiados por el trabajo de la universidad.

Debido al tipo de estudio, para la recolección de datos no sólo se hizo uso de datos secundarios, tales como las evaluaciones numéricas obtenidas a través de estudios estadísticos previamente realizados, sino que fue necesario la utilización del instrumento de la encuesta a fin de poder conocer directamente de las personas que se encuentran dentro de la región de estudio el impacto de la Universidad Intercultural en sus vidas desde el punto de vista económico, social y ambiental. La fiabilidad y validez del instrumento se analizó por el coeficiente Alfa de Cronbach.
El proceso de análisis se realizó mediante el software SPSS cuyo cálculo emplea el promedio de todas las correlaciones existentes entre los ítems del instrumento, con base a los resultados el valor de Alfa de Cronbach obtenido fue de .811 de acuerdo con la escala De Vellis citado por García y Cirilo (2006), esto representa un nivel muy bueno de confiabilidad del instrumento.

\section{Resultados}

La región de la Huasteca se caracteriza por su configuración multiétnica, su heterogeneidad ambiental y su riqueza ecológica; lo que permite la utilización de variados recursos. El uso del suelo de la región se ha destacado por ser predominantemente agrícola y ganadera, esto se ve reflejado directamente en los municipios encuestados donde el nivel educativo de las personas en su mayoría (74.33\%) no han concluido la educación básica o media superior y por consiguiente no cuentan con algún título universitario. La UVI surge en esta región con el objetivo principal de formar profesionales comprometidos con el desarrollo económico y cultural en el ámbito regional, y con la finalidad de que sus actividades contribuyan a fortalecer la región huasteca (Universidad Veracruzana, 2015).

Aunque un $37 \%$ de las personas encuestadas no tenían conocimiento alguno sobre la existencia de la UVI, el $63 \%$ si conocen esta institución e incluso consideran que su presencia en la región de la huasteca es benéfica, puesto que para ellos representa no sólo una forma de conservación de la cultura (ya que la Universidad apoya a los ritualistas a la conservación de la misma), sino también una oportunidad de estudio, y para el $72 \%$, un beneficio a la economía familiar, no sólo porque el costo de la inscripción es relativamente bajo, sino también porque coadyuva a que los hijos no tengan que migrar a otros lugares para continuar con sus estudios, lo que se ha reflejado en reducir los costos educativos.

Lo anterior, se puede ver reflejado en el número de personas que han obtenido el grado de licenciatura en esta región, pues de acuerdo con un estudio de la Universidad Veracruzana Intercultural (2016), del total de personas con grado de licenciatura, el $87 \%$ ha sido a través de la UVI y el $13 \%$ de otros centros de educación superior, como son: 
UPN, ICEST, ITSCHI, ITN, UPAV, UNAL. Paralelamente, en el mismo estudio, se puede encontrar que del total de personas que estudian en la UVI, el 60\% hablan y entienden el náhuatl (lengua originaria indígena), lo que permite ver la importancia que le dan a la preservación de la cultura, junto con el desarrollo de diversos proyectos que permiten dar a conocer las comunidades de la región y fomentar la preservación de las costumbres.

Por otro lado, el 58\% considera benéfica la presencia de la UVI para la comunidad, al representar no sólo una fuente de empleo, si no también, una forma de crecimiento a través del trabajo que realizan sus estudiantes con la comunidad y las personas de esta. Retomando el estudio antes mencionado, se observa que el $82 \%$ de la población entre un rango de edad del 22 a 56 años, es población ocupada, de la cual el $93 \%$ es trabajadora, el $17 \%$ trabaja como independiente (autoempleo) y el 7\% continua con sus estudios (Universidad Veracruzana intercultural, 2016). Asimismo, las profesiones que desarrollan "se ubican preferentemente en el Sector de servicios: Servicios educativos (36\%), servicios administrativos (33\%), servicios culturales (8\%), servicios comunicativos $(8 \%)$, y servicios de salud (5\%)" (Universidad Veracruzana intercultural, 2016: 20). Lo anterior, permite confirmar la opinión de las personas encuestadas.

En cuanto al ingreso que obtienen aquellas personas que egresan de la UVI, se encuentra que el $17 \%$ percibe un ingreso por debajo del salario mínimo, percibiendo en su mayoría ingresos alrededor de \$190 US mensual (Universidad Veracruzana intercultural, 2016). Hay que destacar que, en aquellas comunidades que más cercanas se encontraban a la UVI, reflejaban mayores percepciones positivas, mientras que, en aquellas comunidades más alejadas, las percepciones negativas si no es que nulas, eran mayores; por ejemplo, en el municipio de Benito Juárez, era común una percepción de poco beneficio para las familias, con un porcentaje del $12 \%$, al no entender el objetivo de Universidad dentro de la comunidad. En síntesis, podría decirse que, la UVI ha contribuido de forma positiva a la parte económica y social de la región; dejando entre ver diversos puntos que representan oportunidades para la institución.

\section{Discusión}

Se ha partido de la convicción de que a través del incremento de la formación educativa de una comunidad se contribuye directamente en el desarrollo de los diversos ámbitos de esta, poniendo a la educación superior como el peldaño más alto por alcanzar. De ello deriva que, dentro de los objetivos primordiales de un gobierno, se encuentre el propósito de abarcar los diferentes sectores que conformen la sociedad. Para contribuir con ello, en México se han ubicado Universidades Interculturales en regiones especialmente necesitadas de procesos de desarrollo, las cuales son culturalmente sensibles, socialmente distributivas y con una ubicación especialmente privilegiada para demostrar la calidad existente.

La discusión debe enfocarse a lo que menciona Ávila y Ávila (2016) en el sentido de que la educación intercultural es una demanda legítima de los pueblos indios de México por dotarse de instrumentos educativos que permitan hacer frente a la discriminación y a los procesos de asimilación que se ejercieron como parte de la política pública en el siglo XX, desafortunadamente menciona que a más de diez años de la creación de estas universidades, somos testigos de cómo las mismas se han subsumido en modelos universitarios tradicionales dentro de la episteme hegemónica y se han ido convirtiendo en focos de control de grupos de poder estatales pese a contar con recursos del gobierno federal, de tal forma que no se está orientando realmente a la interculturalidad sino a adaptar modelos educativos tradicionales que no logran una asimilación de la cultura de los que estudian en una Universidad Intercultural.

La Universidad Veracruzana desde 2005 ha emprendido la tarea de favorecer la diversidad cultural de la sociedad, atendiendo las necesidades de acceso a estudios de educación superior de la población de regiones interculturales. Después del análisis presentado, la discusión debe enfocarse en el sentido de si la UVI cumple el objetivo primordial de la Coordinación General de Educación Intercultural y Bilingüe en México, que es ampliar la proporción de los indígenas en la matrícula de educación superior; actualmente se tienen 724 estudiantes, indicando un aumento del $46.40 \%$ de cuando dio inicio. 


\section{Conclusiones}

Se concluye tomando en cuenta el impacto regional que tiene las dimensiones del desarrollo endógeno que, en cuanto a la parte económica, la UVI ha sido proveedora de conocimiento, el cual se transforma en la mejora continua de proyectos dirigidos al campo, permitiendo obtener mayores ingresos. Los egresados de la UVI han podido ser competitivos en comparación de las personas que habitan en la comunidad.

Es importante resaltar que la economía de los egresados de la UVI, así como la de sus familias, se ha visto mejorada gracias a la inserción laboral que han tenido, tanto en el sector público como en el privado, o en algunos casos han optado por el autoempleo, sin embargo, se debe considerar que una mayor oferta académica, ya que en muchos de estos trabajos puede ser que no se encuentren aplicando los conocimientos adquiridos o que no se encuentre relación con lo que estén desempeñando. La UVI en algunas ocasiones ha sido creadora de fuentes de empleo, teniendo actualmente a 8 exalumnos desempeñando labores administrativas.

Hablando del impacto social-cultural, la UVI se ha caracterizado por realizar eventos para la promoción de la ciencia, tecnología, la cultura y las manifestaciones artísticas; entre los que se pueden mencionar: los museos de la ciencia, el festival del buen vivir, las jornadas interculturales, los intercambios de semillas, los foros sobre políticas municipales, las presentaciones de libros, las conferencias, así como también las celebraciones de día de muertos, las presentaciones de teatro, los Cine club, entre otros, los cuales permiten que niños, estudiantes de secundaria y bachillerato, organizaciones campesinas, artesanos, profesores y autoridades municipales, visiten frecuentemente estos espacios y tengan acceso a tales experiencias.

Además, la UVI ha prestado servicios de atención comunitaria para consulta o asesorías en áreas como la jurídica, salud, educación y ecología.
En cuanto al ámbito ambiental tecnológico, a pesar de que la UVI cuenta con la infraestructura tecnológica para generar proyectos de calidad sustentables, dado que cada sede tiene un laboratorios suficientemente equipados, han existido limitantes para garantizar la frecuencia de actividades de este tipo en las regiones, por ejemplo, la escasez o retardo de la disponibilidad de recursos, la lentitud de los trámites que implica el ejercicio de los recursos, o bien, la falta de dominio por parte de los estudiantes.

Se sugiere atender una de las principales preocupaciones de la UVI que es la manera de entablar relaciones con la sociedad, ya que de no incorporarse a la vida productiva únicamente se quedará como un proyecto de superación escolar, sin ver los beneficios que podría traer; por lo tanto, se propone el fortalecimiento de la difusión de los perfiles de formación de los egresados, para que sean conocidos en todo el país y puedan desarrollarse de manera personal, mejorando su calidad de vida y de la comunidad. Otra situación que se debe poner atención es la forma en que se visualiza desde el exterior el funcionamiento de la UVI, tratando de conseguir el reconocimiento por las aportaciones de las culturas indígenas en el mundo laboral, con el fin de evitar actitudes intolerantes y acciones discriminatorias a las personas pertenecientes a estas comunidades.

Otro aspecto significativo es la valoración y pertinencia de la licenciatura que se imparte, ya que también se recibieron comentarios en el sentido de que los programas educativos deben enfocarse a las necesidades propias de la región, para ello proponen carreras que tengan que ver con la parte agrícola ya que muchos de los que habitan en los municipios y localidades cercanas a la UVI se dedican al cultivo y comercialización de productos agrícolas, para el estudio de este tipo de carreras los hijos de las familias deben abandonar sus hogares lo que genera costos adicionales en la educación y muchos de ellos tienen que truncar sus estudios por la falta de recursos económicos. 
Con base en lo anterior, se considera que la UVI se encuentra en proceso de cumplimiento de los ámbitos que se mencionan dentro del desarrollo endógeno, en este sentido, tal como lo marca la misión y visión de la UVI, se pretende el impulso de una economía alternativa bajo una perspectiva de descentralización y en condiciones óptimas para su funcionamiento, si bien, la UVI ha participado activamente en ello, en la mayoría de los casos le corresponde a los estudiantes asumir son pocos recursos $y$ capacidades los retos del mercado global.

\section{Referencias}

Ávila Romero, L., \& Ávila Romero, A. (2016). Las universidades interculturales de México en la encrucijada. Revista de ciencias sociales y humanidades, 25(50), 200- 215. https://doi.org/10.20983/noesis.2016.2.8

Ávila Romero, Agustín, \& Ávila Romero, León Enrique. (2014). El asalto a la interculturalidad: las universidades interculturales de México. Argumentos (México, D.F.), 27(76), 37-54. Recuperado en 24 de abril de 2020, de http://www.scielo.org.mx/scielo.php?script=sci _arttext\&pid=S0187-

57952014000300003\&lng=es\&tlng=es.

Bula, J. (1994). John Rawls y la teoría de la modernización. Cuadernos de Economía., Vol. 14 (21), p. 67-83 https://revistas.unal.edu.co/index.php/ceconomi a/article/view/14048

Blacutt, M. (2013). El desarrollo local complementario

Bourdieu, P. (1998). Capital cultural, escuela y espacio social ( $2^{\circ}$ ed.). Madrid, España: Siglo XX1 editores S.A. de C.V.

Casillas Muñoz, M. D., \& Santini Villar, L. (2006). Universidad intercultural. Modelo educativo ( $1^{\mathrm{a}}$ ed.). México, D.F.: Coordinación General de Educación Intercultural y Bilingüe.

Delgado, F. (2010). El desarrollo endógeno sustentable: como interfaz para implementar el vivir bien en la gestión pública boliviana. Primera edición. Bolivia: Agruco.
Diario Oficial de la Federación. (2014). "Plan Nacional de Desarrollo 2013-2018”. Secretaría de Gobernación, en: http://www.dof.gob.mx/nota_detalle.php?codig $o=5299465 \&$ fech $a=20 / 05 / 2013$

Freire, P. (1970). La Educación como práctica de la Libertad. Montevideo, Uruguay: Tierra Nueva.

García Cadena, Cirilo H. (2006). La medición en ciencias sociales y en la psicología. Estadística con SPSS y metodología de la investigación. México. Trillas

Giroux , H. (1992). Teoría y resistencia en educación. Buenos Aires, Argentina: Siglo XX1. Guitart, M. E., \& Rivas, M. J. (2008). La propuesta de las Universidades Interculturales en México frente al pluralismo cultural: El caso de Chiapas. Documentación social(151), 147162.

Huntington, S. (2006). The change to change: Modernization, Development, and Politics. http://home.ku.edu.tr/ dyukseker/huntingtonchangetochange.pdf

Kotler, P., Jatusripitak, S., \& Maesincee, S. (1998). El Marketing de las Naciones. En Una aproximacion estratégica a la creación de la riqueza nacional. (págs. 122-124). Barcelona: Paidos.

Mato, D. (2009). Instituciones Interculturales de Educación Superior en América Latina. Panorama Regional, Procesos Interculturales de Construcción Institucional, Logros, Dificultades, Innovaciones y Desafíos. En D. Mato, Instituciones Interculturales de Educación Superior en América Latina. Procesos de Construcción, Logros, Innovaciones y Desafíos. Caracas: Iesalc-Unesco.

Morán, E., La economía comunal. Edición electrónica gratuita. Obtenido de www.eumed.net/libros/2007c/335/

Muñoz, C. (2014) El papel de la educación en el desarrollo económico y social; una perspectiva http://resu.anuies.mx/archives/revistas/Revista3 7_S1A2ES.pdfNorth, D. C. (1990): Instituciones, cambio institucional y desempeño económico. Fondo de Cultura Económica, México, 1993. 
Muñoz, C. (2014). Relaciones entre educación y justicia. Revista Latinoamericana de Estudios Educativos. México.

Reyes, G. (2001). Principales teorías sobre el desarrollo económico y social. Revista crítica de ciencias sociales y jurídicas.

Romero Ávila, A. (2011). Universidades interculturales y colonialidad del saber. Revista Educación y Desarrollo (16), 20-25.

Sen, A. (2001): Development as Freedom, Nueva Delhi, Oxford, Oxford University Press, 2. ${ }^{\mathrm{a}}$ edición.

Schwartzman, S. (2005). Educación y pobreza en América Latina. Diálogo Politico (4), 11-40 Polanco, E. (9 de noviembre de 2012). Veracruz Vive. Obtenido de División Política y municipios:

http://veracruzvive.blogspot.mx/2012/11/divisio n-politica-y-municipios-de.html

Polanyi, K., Arensberg, M. C. y Pearson, W. H. (1957): Trade and Market in the Early Empires: Economies in History and Theory. Chicago, Henry Regnery Company.

Tito, J. (2012). Modernización e integración del derecho contractual. Un análisis del método. Primera edición: Editorial Universidad del Norte Editorial.

UNESCO. (2017). Educación $e$ Interculturalidad. Recuperado el 2017, de Organización de las Naciones Unidas para la Educación, la Cienia y la Cultura: http://www.unesco.org/new/es/quito/education/ education-and-interculturality/

Universidad Veracruzana. (2007). Licenciatura en Gestión Intercultural para el Desarrollo. Programa Multimodal de Formación Integral. Obtenido De Universidad Veracruzana Intercultural:

https://www.uv.mx/uvi/files/2012/11/2007_LGI D_DEx.pdf

Universidad Veracruzana. (2016). Licenciatura en Derecho con enfoque de Pluralismo Jurídico. Plan de estudios 2016. Obtenido de UVIntercultural:

https://www.uv.mx/uvi/files/2012/11/160212_L DEPLUJ_v9.pdf
Universidad Veracruzana. (2015). $\quad U V$ Intercultural. Obtenido de Proyecto: http://www.uv.mx/uvi/proyecto-intercultural/ Universidad Veracruzana intercultural. (2016). Reporte de situación laboral y necesidades formativas de egresados 2015. Obtenido de UNIVERSIDAD VERACRUZANA INTERCULTURAL:

https://www.uv.mx/uvi/files/2016/03/UVI_Repo rteEgresados_2015.pdf

Universidad Veracruzana. (2017). UVIntercultural. Obtenido de Proyecto: http://www.uv.mx/uvi/proyecto-intercultural/ Vásquez, A. (2007). Desarrollo endógeno y globalización.

http://www.redalyc.org/pdf/196/19607903.pdf 